\title{
Zum Problem der saaleeiszeitlichen Terrassenbildungen im Unterlauf der Werre
}

\author{
Von J.-H. Henke, Rotenhagen \\ Mit 4 Abbildungen
}

$\mathrm{Z}$ u s a m menfassung. Die problematische Stellung der saaleeiszeitlichen Terrassenablagerungen der Werre, die sich aus der einschlägigen Literatur und den Geologischen Karten ergibt, veranlaßte den Verf. zu Terrassenuntersuchungen am Unterlauf der Werre bei Gohfeld und Bad Oeynhausen. Vor allem wurden der stratigraphische Aufbau der Ablagerungen untersucht und Rundungsmessungen an Quarzkörnern vorgenommen.

Als Beispiel wird die Terrasse bei Gohfeld, Nähe Bahnhof, an der eiszeitlichen Einmündung des Sudbaches in die Werre näher beschrieben und klimatisch gedeutet.

Im Hangenden des Terrassenkörpers befinden sich solifluidaler Wanderschutt bzw. Niederterrassenablagerungen, im Liegenden saaleeiszeitliche Sande und Kiese mit tieferreichenden Froststrukturen.

Bei den Kiesen handelt es sich um nordische Feuersteine; die Porphyre und Granite stammen aus dem Thüringer Wald und sind daher ursprünglich von der Weser herantransportiertes, mehrfach umgelagertes Material. Die überwiegende Mehrzahl der Quarzkörner ist mittelmäßig gerundet und auf jeden Fall transportbeansprucht.

Für eine einheitliche Fließgeschwindigkeit des Flusses und Akkumulation im Mittelterrassenkörper spricht die relativ gute Sortierung, denn Anzeichen für Staubeckenbildungen wurden bisher nicht gefunden. Zu Beginn der Saaleeiszeit lag die Talsohle der Werre bei Gohfeld im Lias etwas über dem Niveau der heutigen Talaue.

Die Anknüpfungen der Terrassen des Werreunterlaufs an die saaleeiszeitlichen Flußablagerungen im Wesersystem konnten festgestellt werden.

$\mathrm{Summary}$. The classification of the fluviatile deposits of the river Werre showing its problematic character in the literature on the subject, caused the author to study the terraces of the lower course of the Werre near Gohfeld and Bad Oeynhausen.

The stratigraphy of the layers and the roundings of quartz grains were taken into special consideration.

By way of example the terraces near the station of Gohfeld are closely described and examined according to climatic changes during the ice age:

Two different horizons could be distinguished:

1) Keuper débris caused by solifluction, fluviatile deposits of the Lower Terrace respectively,

2) sands and pebbles of the Saale glacial epoch with periglacial frost-structures.

The pebbles consisted of Scandinavian flints; the porphyries and granites are originated in the Thüringer Wald.

Most of the quartz grains have a moderate rounding, proving in any case that they were transported by water.

The sorting is relatively good and indicates a uniform flowing speed of the river and accumulation in a Middle Terrace, for deposits in slack water could not be detected.

In the early Saale the bed of the river Werre was at the level of the recent bottom of the valley, reaching Lias.

The reference to the Middle Terrace of the river Weser could be stated.

Die Bestimmung der saaleeiszeitlichen Sand- und Kiesablagerungen im Gebiet der Werre gestaltet sich aus folgenden Gründen recht schwierig: Die Schmelzwasserablagerungen des Inlandeises liegen über und unter der Grundmoräne und scheinen sich nicht von den Sanden der Niederterrassen zu unterscheiden. Auch morphologisch ist die Abgrenzung der älteren eiszeitlichen Ablagerungen zu denen der Niederterrassen undeutlich. Die Terrassenränder steigen zu den Hängen meist kontinuierlich an, so daß eine Einordnung der Terrassen nur nach morphologischen Gesichtspunkten kaum möglich ist. Leider 
wurden auch keine interglazialen Ablagerungen festgestellt, auf Grund derer man eine Gliederung hätte vornehmen können.

Die problematische Stellung der saaleeiszeitlichen Terras s e n bildungen spiegelt sich auch in der einschlägigen Literatur wider: MESTWERDT (1922, S. 29), der sich sehr um die Bestimmung der älteren eiszeitlichen Ablagerungen bemühte, stellte in den Erläuterungen zum Geologischen Blatt Herford-Ost, Nr. 2083 folgendes Gesamtprofil der Entwicklung des Taldiluviums der Werre auf:

(Jüngste) Eiszeit: Aufschüttung der Unteren Terrasse,

Letzte Interglazialzeit: Ausfurchung in zwei Talstufen von verschiedener Höhenlage,

(Mittlere) Eiszeit: Mischung der Vorschüttsande mit Schottern einheimischer Gesteine.

Driever (1921, S. 79) setzt die 20-, 5- und 2-m-Terrassen im Werre-Else-System zu den saaleeiszeitlichen Schotterablagerungen an der Porta in Beziehung und bezeichnet sie als postglazial.

Auf der Geologischen Karte von Bad Oeynhausen, im Maßstab $1: 25$ 000, Nr. 3718 (hrsg. 1938), die von Koert sowie Stach bearbeitet und von Dienemann ergänzt wurde, werden im Bereich der Werre eine Untere Terrasse der letzten Eiszeit (Delta 3s) und Schmelzwasserablagerungen der vorletzten Eiszeit (ds) kartiert. In den Erläuterungen zu den Geologischen Blättern Melle, Quernheim und Oeynhausen von Dienemann (1939, S. $49 \mathrm{ff}$ ) werden zwar saaleeiszeitliche Ablagerungen näher beschrieben, eine Mittlere Werreterrasse wird jedoch nicht erwähnt. Auch ZIERCKE (1960, S. 51) verzeichnet an der Werre keine Mittlere Terrasse, wobei allerdings zu beachten ist, daß ältere Sande und Kiese im Unterlauf der Werre durchaus diskutiert wurden (S. $75 \mathrm{ff}$ ).

In der Literatur werden also die eiszeitlichen Ablagerungen der Werre verschieden gedeutet.

Der folgende Beitrag versucht, das Problem der älteren Sande und Kiese am Nordhang des „Herforder Keupervorsprunges" zur Liasmulde hin zwischen Gohfeld und Bad Oeynhausen durch Untersuchungen hinsichtlich des stratigraphischen Aufbaus der Ablagerungen und Rundungsmessungen an Quarzkörnern zu lösen.

Für die sedimentpetrographischen Analysen ist der Verf. den Herren Direktor Dr. LütTig und Dr. MatTiat vom Niedersächsischen Landesamt für Bodenforschung in Hannover zu Dank verpflichtet (vgl. Abt. Nr. 60 769-60 770).

Nach vorläufiger Aufnahme zieht sich mit nur kleinen Unterbrechungen ein einheitlich aufgebauter Terrassenkörper von Gohfeld bis Bad Oeynhausen entlang der Bahnlinie Bielefeld-Minden hin. Seine Ausdehnung fällt etwa mit der Fläche zusammen, die auf der Geologischen Karte Oeynhausen, Nr. 3718, als ds bzw. dm ausgeschieden wurde. Die Geländeoberfläche der Terrasse liegt bei Gohfeld $57,5 \mathrm{~m}$, bei Bad Oeynhausen am Mittelbach 56,5 m über NN. Auf einer Strecke von 1,5 km Luftlinie entspricht das Gefälle im Terrassenkörper mit ca. $1 \mathrm{~m}$ dem in der Talsohle der Werre gemessenen Wert. Die Mächtigkeiten der Terrassenablagerungen betragen bei Gohfeld mehr als $3 \mathrm{~m}$, im Aufschluß am Mittelbach mindestens $6 \mathrm{~m}$, denn der Liasuntergrund wurde hier nicht erreicht.

Als Beispiel wird der Terrassenkörper an der weichseleiszeitlichen Einmündung des heutigen Sudbaches in die Werre ( $\left.\mathrm{H}: 52^{\circ} 12^{\prime} 12^{\prime \prime}, \mathrm{R}: 8^{\circ} 45^{\prime} 30^{\prime \prime}\right)$ näher beschrieben und klimatisch gedeutet. Er liegt ca. 7-10 m über der Talaue der Werre und wird von der Bahnlinie Löhne/W.-Bad Oeynhausen durchschnitten.

Die Bestimmung des Vereisungsalters durch Gesteinsanalyse der Kiese mußte scheitern, da die Ablagerungen außer Feuersteinen kein nordisches Material aufwiesen. Von kristallinen Gesteinen konnten Porphyre und Granite aus dem Thüringer Wald nachgewiesen werden (briefliche Nachricht von Prof. Dr. Hesemann, Krefeld, v. 6. 1. 68). Dies ist auch nicht weiter erstaunlich, da selbst in den Kamesbildungen von Hausberge/Porta Westfalica nordisches Material nur spärlich verteilt ist. 
Der stratigraphische Aufbau der oben erwähnten Terrasse am Sudbach ergab folgendes Bild:

$10 \mathrm{~cm}$ humoser sandiger Lehm

$70 \mathrm{~cm}$ Schwemmlöß, der nach unten immer mehr in Sand übergeht und auch eckiges Geröll, z. B. Granite und Feuersteine, führt

$70 \mathrm{~cm}$ kantig-plattige, horizontal geschichtete Lokalschotter aus Keuper

3,20 m teilweise kreuzgeschichtete Sande mit kleinen Splittern aus nordischen Feuersteinen, abgeplatteten Sandsteinen sowie zahlreichen schwärzlichen Schiefertonschüppchen, die aus dem Lias stammen dürften, der im Liegenden der Sande ansteht.

In die Sande und Schotter reichte eine Frostspalte von 1,40 m Tiefe und $40 \mathrm{~cm}$ oberer Breite (Abb. 1).

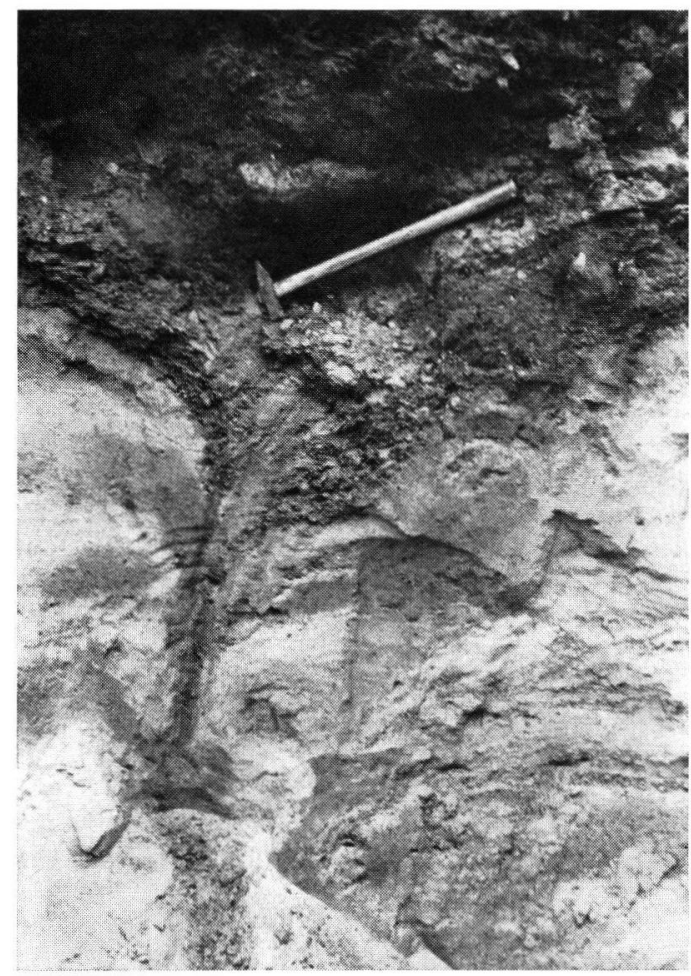

Abb. 1. Frostspalte, die bis in die Mittelterrassensande und -kiese der Werre bei Gohfeld, Nähe Bahnhof, reicht.

Deutlich erkennt man, daß sich die Ablagerungen aus zwei verschieden strukturierten Schotterkörpern zusammensetzen: Dem Schotterkörper im Hangenden und den liegenden Sanden und Kiesen, zwischen denen offenbar eine längere Abtragungsperiode gelegen hat, wie es ein Aufschluß $150 \mathrm{~m}$ weiter nördlich im gleichen Terrassenkörper andeutet, wo an Stelle des Keuperschotterhorizontes im Hangenden sich diskordant Sande mit Keupergeröllen befinden. Für die Ablagerung der beiden Schotterkörper machen wir ein differenziertes Klima verantwortlich.

Bei den hangenden Partien handelt es sich um Wanderschutt mit fluviatiler Überprägung, wie es auch die Indizes mit 48\% über Index-Wert 100 zeigen. Am Mittelbach bei Bad Oeynhausen liegt das Maximum mit 64\% bei den Indizes 50-100 (Henke 1968, S. 70). Die fluviatile Tätigkeit der Werre kann nicht als Ursache für die Überformung 
angeführt werden. Der Wanderschutt wird sich in einem Klima mit Dauerfrostboden gebildet haben, wie es aus dem Frühglazial der Weichseleiszeit bekannt ist. Der Schutt wurde im vorliegenden Fall durch den Sudbach transportiert und lagerte sich dann an dessen eiszeitlicher Einmündung in die Werre ab. Er gelangte von den Keuperhängen in den Ober- und Mittellauf des Baches, denn im Unterlauf des Sudbaches steht Lias an. $\mathrm{Daß}$ es sich nicht um saaleeiszeitlichen Wanderschutt handeln kann, wird durch die Tatsache erklärt, daß dieser bei dem folgenden Gletschervorstoß vernichtet worden wäre. Auch die Frostspalte, die sich durch diesen Schuttkörper bis in die saaleeiszeitlichen Sande und Kiese erstreckt, hätte zerstört werden müssen oder dürfte gar nicht erst zur Ausbildung gekommen sein. Aus der erhalten gebliebenen Froststruktur, die im extrem-kalten Klima des Hochglazials der letzten Eiszeit gebildet wurde (Henke 1968, S. 55 f), können wir gleichfalls schließen, daß die Schuttbildung im Frühglazial erfolgte. Wenn aber für den Keuperschuttkörper weichseleiszeitliches Alter anzunehmen ist, müssen die liegenden sandig-kiesigen Ablagerungen älter sein und der vorletzten Eiszeit angehören, denn interglaziale Bildungen konnten nicht festgestellt werden.

Zwei Sandproben aus dem als Beispiel genauer beschriebenen Terrassenkörper bei Gohfeld repräsentieren einen schwach schluffigen grobsandigen Mittelsand mit nur geringen Kiesanteilen. Die Sortierung ist relativ gut, bei Probe $1(0,50 \mathrm{~m}$ Tiefe) etwas schlechter als bei der zweiten Probe (1,50 m Tiefe). Die Probe 1 enthält etwas mehr Schluff, Grobsand und Kies (Abb. 2).

Der Abrollungsgrad wurde an Quarzkörnern in den Fraktionen 0,2-0,63 mm und 0,63-2 mm Durchmesser bestimmt. Die überwiegende Mehrzahl der Körner liegt in den Rundungsklassen III und IV nach DAL VESCO, d. h., daß die Quarzkörner mittelmäßig gerundet und damit auf jeden Fall transportbeansprucht sind.

Im Gegensatz zu den Sandproben von Falkendiek, Meßtischblatt Herford-Ost, Nr. 2083, spricht die bessere Sortierung im Unterlauf der Werre für eine einheitliche Fließgeschwindigkeit und Akkumulation des Flusses im Mittelterrassenkörper. Anzeichen für Staubeckenbildungen, wie im Hameln-Rintelner Stausee an der Weser, konnten bisher nicht entdeckt werden. Nach einer brieflichen Mitteilung von Dr. habil. Wor tmann, Göt-

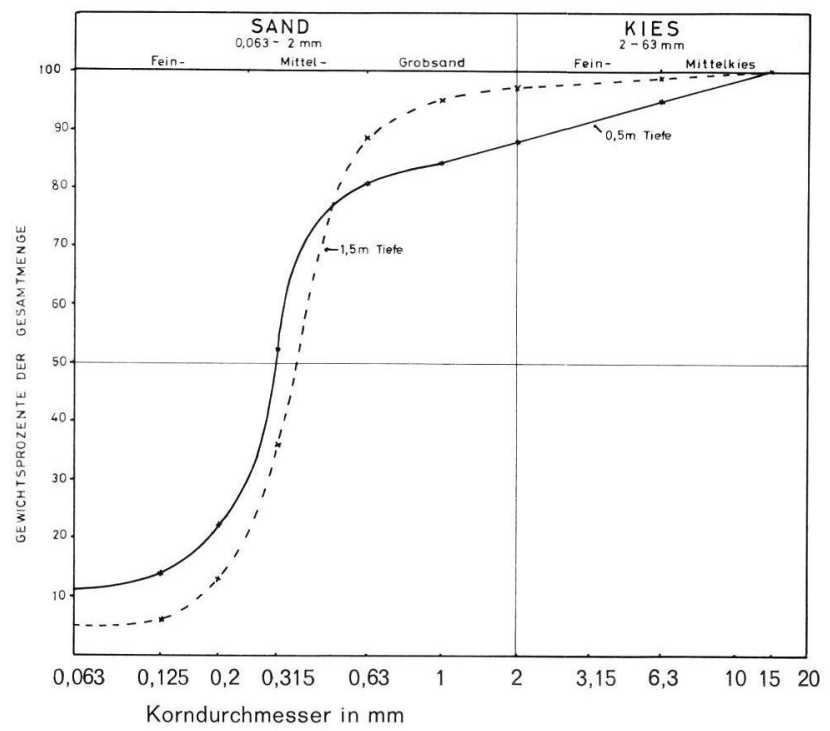

Abb. 2. Korngrößenverteilung der Mittelterrassensande und -kiese bei Gohfeld, Nähe Bahnhof. 0,50 m Tiefe, - - - 1,50 m Tiefe. 
tingen, sind die Beckentone, die die Wesermittelterrasse bedecken, bis zum Bahnhof Oeynhausen-Süd nachgewiesen.

Bei Baugrunduntersuchungen für die Widerlager der Fußgängerbrücke über den 1966 neuangelegten Werredurchstich im Sielpark des Staatsbades Oeynhausen (Nähe Bülow Brunnen) stellte man unmittelbar unter der Sohle des neuen Werrebettes im Durchstich Tonschiefer des Lias in unbekannter Mächtigkeit fest. Dies teilte Kreisbauoberinspektor Mietrup aus Minden dem Verf. am 15. 11. 1967 brieflich mit. Im Hangenden der Tonschieferschicht wurden bis zur Geländeoberfläche ca. $6 \mathrm{~m}$ mächtige Sande beobachtet, die mit leicht bindigen Bestandteilen durchsetzt waren (Abb. 3).
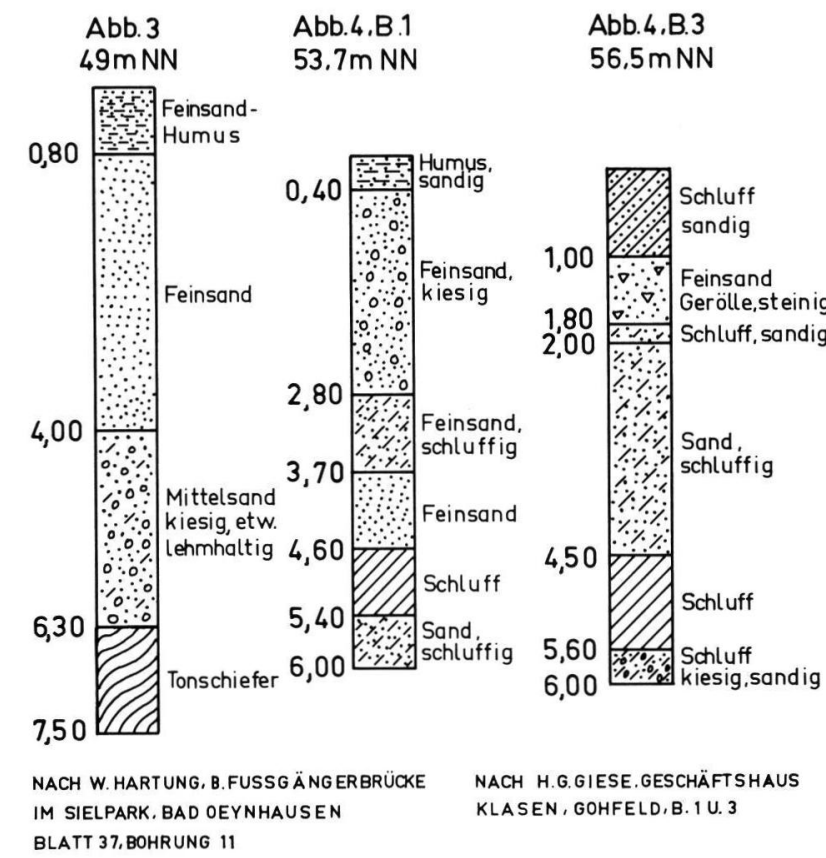

Abb. 3 u. 4. Die Schichtenfolge in 3 Bohrprofilen (Maßstab der Höhe $1: 50$ ).

$\mathrm{Da}$ für das saaleeiszeitliche Spätglazial Abtragung angenommen werden muß, werden die Sande und Kiese der Werre bei Gohfeld im Frühglazial oder zu Beginn des Hochglazials zur Ablagerung gekommen sein. Dies kann auch aus der Überlagerung der unter gleichen Bedingungen sedimentierten Sande mit Grundmoräne des Drenthe-Stadiums in einem Salze-Aufschluß, Nähe Gehöft Möller, Exter, geschlossen werden (Geolog. Karte Herford-Ost, Nr. 2083). Bei Gohfeld liegen auf den seitlichen Hängen nur noch Steinsohlen als Reste einer ehemals viel mächtigeren Grundmoräne. Die Talsohle der Werre lag zur Zeit der Ablagerung der Sande, also zu Beginn der Saaleeiszeit, im Lias hier ca. 1-2 $\mathrm{m}$ über dem Niveau der heutigen Talaue der Werre $(50 \mathrm{~m}$ über $\mathrm{NN}$ bei Starke an der Werrebrücke). In ähnlicher Weise blieben auch die Schotterkörper, die sich zur Weichseleiszeit über die älteren Sande und Kiese legten, an den Einmündungen der größeren Bäche, wie Mittel- und Osterbach, in die eiszeitliche Werre erhalten (Abb. 4), nur daß die Sande und Kiese hier bereits unter das Niveau der heutigen Talsohle der Werre reichen. Zum Werrefluß selbst hin liegen die Basiswerte der saale- bzw. elstereiszeitlichen Flußablagerungen wesentlich tiefer, und zwar $+41 \mathrm{~m}$ NN am neuangelegten Werredurchstich im Sielpark von Oeynhausen. An der Einmündung der Werre in die Weser bei 
Rehme gibt Ziercke (1960, S. 83) +34 m NN als Niveau der elstereiszeitlichen Werre an. Seitdem ist das Werretal ca. $10 \mathrm{~m}$ höher gelegt worden. Wie im benachbarten Wesersystem reichen offenbar die älteren Flußablagerungen der Werre tiefer als die der weichseleiszeitlichen fluviatilen Ablagerungen.

Die Deutung des oben ausführlich beschriebenen Terrassenkörpers als Mittelterrasse wird ferner unterstützt durch die Beziehung zur Wesermittelterrasse. Die Oberkanten der Mittelterrassen liegen bei Gohfeld $56 \mathrm{~m}$, am Mittelbach/Oeynhausen 55,5 über NN. Nehmen wir mit Ziercke (1960, S. 83) an, daß das Gefälle im Unterlauf der Werre seit der Elstereiszeit bis heute ziemlich gleich geblieben ist, so dürfen wir für die Strecke GohfeldRehme ca. $4 \mathrm{~m}$ Gefälle ansetzen. Im Mündungsgebiet der Werre in die Weser müßte die Oberkante der Mittelterrasse also ca. $51 \mathrm{~m}$ über NN liegen, d. h. minimal $7 \mathrm{~m}$ über der jetzigen Talsohle. Wir befinden uns demnach im Niveau der Wesermittelterrasse bei Holzhausen/Porta.

\section{Literaturund Karten}

W. Dienemann: Erläuterungen zu den Blättern Melle, Quernheim, Oeynhausen, 145 S., Berlin 1939.

E. Driever: Die Entwicklung des Längstales Porta-Osnabrück. - Jahresber. d. Naturw. Ver. zu Osnabrück, 18, 1-88, Osnabrück 1921.

J.-H. HenkE: Morphologie des Herforder Keupervorsprunges unter besonderer Berücksichtigung eiszeitlicher Überformung. — Math.-Nat. Diss., 89 S., Hamburg 1968.

A. Mestwerdt: Erläuterungen zur Geologischen Karte von Preußen, Blatt Herford-Ost, Nr. 51, 45 S., Berlin 1922.

I. ZiERCKE: Talentwicklung und Oberflächenformen im Einzugsgebiet der Werre zwischen Teutoburger Wald und Wiehengebirge. - Forsch. z. Dt. Landeskunde, 116, 92 S., Bad Godesberg 1960.

Geologische Karten: Maßstab 1:25000 Bad Oeynhausen Nr. 3718, Herford-Ost Nr. 2083.

Manuskr. eingeg. 26. 2. 1969.

Anschrift des Verf.: Oberstudienrat J.-H. Henke, 4801 Rotenhagen Nr. 28. 